\section{ORIGINAL RESEARCH}

B. Gao

M.I. Baharoglu

A.D. Cohen

A.M. Malek

\title{
Stent-Assisted Coiling of Intracranial Bifurcation Aneurysms Leads to Immediate and Delayed Intracranial Vascular Angle Remodeling
}

BACKGROUND AND PURPOSE: Wide-neck bifurcating aneurysms are increasingly treated with intracranial stent-assisted coiling by using shape-memory alloy microstents. We sought to investigate the short- and long-term effects of intracranial stent implantation on the geometry and angular conformation of the stent-coiled vascular bifurcation.

MATERIALS AND METHODS: Thirty patients underwent stent-mediated coiling for 31 bifurcation aneurysms by using 31 self-expanding Neuroform $(n=14)$ and Enterprise $(n=17)$ stents (17 women; mean age, 56 years). The angle ( $\delta$ ) between the stented mother and daughter vessels at the bifurcation was measured by using multiplanar imaging of reconstructed rotational conventional angiography volumes and was compared by using matched-pair statistics. Neuroform and Enterprise longitudinal stent stiffness was measured in vitro at an increasing bending angle $\theta\left(\theta=180^{\circ}-\delta\right)$.

RESULTS: Stent deployment increased the bifurcation angle $\delta$ from $101.5^{\circ}$ to $119.8^{\circ}$ postprocedurally and to $137.3^{\circ}(P<.0001)$ at latest follow-up, resulting in effective straightening; the angular remodeling was greater in distal-versus-proximal arteries (anterior cerebral $>$ MCA $>$ BA $>$ ICA), inversely proportional to mother-vessel diameter and proportional to pretreatment bending angle $\theta$. At follow-up, angle $\delta$ continued to significantly expand, with remodeling being greater in the early period (1-6 versus $>7$ months) and more pronounced with the longitudinally stiffer closed-cell Enterprise compared with the open-cell Neuroform stent.

CONCLUSIONS: Stent placement across bifurcation aneurysms leads to a significant biphasic angular remodeling related to stent type and vessel caliber, altering morphology to mimic sidewall lesions, a phenomenon needing consideration during procedural planning. Future work is needed to uncover the hemodynamic implications of this structural change and any possible effect on aneurysm-recurrence rates.

ABBREVIATIONS AcomA = anterior communicating artery; $\mathrm{BA}=$ basilar bifurcation aneurysm; $\mathrm{SEM}=$ standard error of the mean, $3 \mathrm{DRA}=3 \mathrm{D}$ rotational angiography

$\mathbf{T}$ he stent-assisted coiling technique has gained increased utility in the endovascular treatment of wide-neck cerebral aneurysms that pose a challenge to conventional coiling because of poor dome-to-neck ratio. ${ }^{1-7}$ The deployment of an intracranial microstent serves as a metal scaffold to prevent coil herniation through the neck of these wide-neck aneurysms and can also increase the treatment - coil packing attenuation. ${ }^{8}$ Since the introduction of stent-placement techniques for aneurysm treatment, significant progress has led to their use in a variety of methods, including sole stent placement for side wall or fusiform aneurysms, ${ }^{4,9-11 ~ " k i s s i n g " ~ s t e n t s ~ f o r ~ w i d e ~}$ fusiform aneurysms, ${ }^{12}$ overlapping stents with or without coiling for ruptured dissecting aneurysms or small wide-neck aneurysms, ${ }^{13,14}$ stents deployed in "Y"15,16 or "waffle-cone"17

\section{Received March 16, 2011; accepted after revision July 13.}

From the Cerebrovascular and Endovascular Division, Department of Neurosurgery, Tufts Medical Center and Tufts University School of Medicine, Boston, Massachusetts.

This work was supported by NIH-R21HL102685 grant.

Please address correspondence to Adel M. Malek, MD, PhD, Department of Neurosurgery, Tufts Medical Center, 800 Washington St, 178 Proger 7, Boston, MA 02111; e-mail: amalek@tuftsmedicalcenter.org

Indicates open access to non-subscribers at www.ajnr.org

http://dx.doi.org/10.3174/ajnr.A2841 configurations, and transcirculation ${ }^{18}$ horizontal stent placement across the neck of a bifurcation aneurysm. ${ }^{19,20}$

Despite the availability of intracranial stent placement for $>7$ years, there remains little information regarding the effect of the deployment of self-expanding microstents (Neuroform, Boston Scientific, Natick, Massachusetts; Enterprise, Cordis, Miami Lakes, Florida) on cerebral vascular architecture. In-stent stenosis has been documented as a low-risk phenomenon in certain cases. $^{21}$ Data on other structural changes following the stentmediated coiling procedure remain sparse. Little information exists on the effect of the deployment of self-expanding stents across vascular bifurcations during stent-mediated coiling of bifurcation aneurysms and specifically on immediate and long-term bifurcation angular configurations. Bifurcation aneurysm stentmediated coiling with a single stent is a multistep process. It involves the deployment of the intracranial stent across the bifurcation over the aneurysm neck from the mother vessel to 1 of the daughter vessels that is most likely to result in best neck coverage and facilitate aneurysm coiling with the least chance of coil herniation or impingement of the other daughter vessel. Recent work has suggested an important contribution to vessel angle with respect to aneurysm inflow. ${ }^{22,23}$ After noting striking cerebral vascular deformation with stent placement in a preliminary analysis, ${ }^{24}$ we sought to investigate the geometric consequences of intracranial stent coiling at bifurcations harboring aneurysms. 


\section{Materials and Methods}

\section{Patient Population and Treatment Method}

Between March 2004 and January 2011, 30 patients (17 females and 13 males) with age range of 13-82 years (mean, 56 years) underwent adjunctive coiling to treat 31 wide-neck bifurcation aneurysms by using intracranial nitinol self-expanding stent assistance with the open-cell design, Neuroform in 14 and the closed-cell design Enterprise in 17. Stent-mediated coiling was offered instead of simple coiling only when endovascular treatment could not be performed without stent assistance and the patient declined surgical clipping of an aneurysm thought to constitute a good risk-benefit ratio for protection from rupture. Patients presented with headache in 5, acute subarachnoid hemorrhage in 7 , recurrence of previously treated aneurysms in 2, vertigo in 1 , and ischemic stroke in 1 case. The aneurysm was found incidentally in 14 patients. There were no cases of hydrocephalus in these patients. One patient with a high-grade subarachnoid hemorrhage had a large hematoma in the right Sylvian fissure, for which he underwent evacuation followed by stent-mediated coil embolization of the aneurysm. The aneurysms were at the following locations: the BA bifurcation ( $n=13)$, the MCA bifurcation $(n=7)$, the ICA bifurcation $(n=3)$, and the anterior cerebral artery bifurcation or AcomA $(n=8)$. Aneurysm size ranged from 2 to $19 \mathrm{~mm}$ (mean $8.6 \mathrm{~mm}$ ), and neck, from 3 to $10 \mathrm{~mm}$ (mean, $6 \mathrm{~mm}$ ).

No intraprocedural aneurysm rupture occurred during any point in the stent-coiling procedures in any of the patients. All stent-coiled patients routinely underwent high-resolution catheter 3DRA before and after stent-coiling and at each angiographic follow-up. This study was approved by the Tufts Medical Center institutional review board (study 9584, entitled "Retrospective Review of Intracranial Cerebrovascular Lesions”).

\section{Stent-Assisted Coiling Procedure and Angiographic Follow-Up Protocol}

Patients underwent interventions under general anesthesia, with dual antiplatelet inhibition with clopidogrel (75 mg/day) and aspirin (acetylsalicylic acid, $325 \mathrm{mg} /$ day) at least 3 days prior and a weight-based intravenous heparin bolus to achieve an activated clotting time of $\geq 240$ seconds before guide-catheter placement. The stents were deployed according to instructions for use of the manufacturer. The targeted aneurysm was accessed with a steam-shaped 150-cm 0.014inch microcatheter (Excelsior SL-10, Boston Scientific) by using the jailing or sequential technique.

The method of image acquisition for stent-mediated embolizations consists of the following: A preprocedural 3D angiogram is obtained for aneurysm measurement and determination of the working projection. A poststent deployment 3D angiogram is obtained to document appropriate deployment, rule out stent migration after delivery-microcatheter withdrawal, and circumferentially rule out a filling defect indicative of stent thrombosis. Finally, a posttreatment 3D angiogram is obtained to evaluate branch occlusion, to rule out filling defects, to assess the relative position of the coils/stent, and for baseline comparison with subsequent imaging to evaluate recurrence or in-stent stenosis.

Patients were followed up both angiographically and clinically at 3-6 months following the stent-deployment procedure, and on the basis of the angiographic findings, the second follow-up angiography was performed at 6 months to 1 year thereafter. The patients also underwent 3T MRA to evaluate the status of the treated aneurysms, and if positive findings were present, digital subtraction angiography was performed for further evaluation or possible retreatment. Follow-up angiography was performed from 1 to 33 months after the initial stent-coiling procedure. Thirty patients completed the first angiographic or MRA follow-up from 1 to 7 months (mean, 3.6 months) after the procedure, 14 patients underwent the second angiographic follow-up from 5 to 31 months (mean, 11.3 months) after treatment, and 5 had a third follow-up from 9 to 33 months after the procedure.

\section{D Modeling and Vascular Measurement}

Biplane 2D digital subtraction angiography and 3DRA imaging were performed on the same flat-panel Axiom Artis system (Siemens, Erlangen, Germany) in each case to assess any branch occlusion, thrombus formation, degree of aneurysmal occlusion, and stent position. The 3DRA imaging in each patient was reconstructed as per the instructions of the manufacturer. The $3 \mathrm{D}$ volumetric datasets were then exported and analyzed by using Amira software (Version 4.1.2, Visage Imaging, San Diego, California) for 3D visualization and angle measurement. The volumetric dataset of each patient was rendered in $3 \mathrm{D}$ space and visualized by using multiplanar reconstruction, orienting it so that the target vascular bifurcation angle $\delta$ (Fig 1 ) was measured at pretreatment, post-stent coiling, and at each angiographic follow-up study by using the same cut-plane. Using orthogonal planes, we measured the diameter of the mother and daughter vessels after applying a Sobel edge-detection filter in 3D space to avoid windowing-related measurement errors. ${ }^{25}$

\section{Force-Angular Deflection Assessment of Stent Longitudinal Stiffness}

Enterprise $(4.5 \times 22 \mathrm{~mm})$ and Neuroform $(4.5 \times 20 \mathrm{~mm})$ stents were secured on their proximal one-third with a $4.5-\mathrm{mm}$ mounting rod, their distal tip deflected at given angle values from $0^{\circ}$ to $65^{\circ}$, and the resulting tangential force measured in grams by using a high-resolution Mettler analytic balance (.0001-g resolution) (Mettler-Toledo, Columbus, Ohio). Orthogonal digital photography was performed at each sample point and was used to measure the effective bending angle $\theta$ and corresponding force for each stent by using an Enterprise and a Neuroform stent. Longitudinal bending stiffness was derived by linear-regression fit.

\section{Statistics}

Vascular angles were compared by use of matched paired $t$ tests by using JMP software, Version 5.0 (SAS Institute, Cary, North Carolina). Univariate and multivariate linear regression analysis was used to assess the association between angular change and various parameters. Statistical significance was assumed at a value of $P<.05$.

\section{Results}

\section{Stent Placement Alters Intracranial Bifurcation Angle in a Biphasic Manner}

During evaluation of angiographic results of aneurysm stent coiling and comparison with follow-up, changes in bifurcation angle $\delta$ were detected angiographically (Fig 1), suggesting that stent deployment led to straightening of the vessel bifurcation. The stented angle $\delta$ at the bifurcation was measured before and after stent placement and at every follow-up (Table). Stent coiling resulted in a highly significant increase in bifurcation angle $\delta$ from a pretreatment value of $103.0^{\circ}\left(41^{\circ}-\right.$ $\left.140^{\circ}\right)$ to $121.0^{\circ}\left(52^{\circ}-155^{\circ}\right)$ posttreatment $(P<.0001)$, further increasing to $137.3^{\circ}$ at latest follow-up $(P<.0001$ versus both 

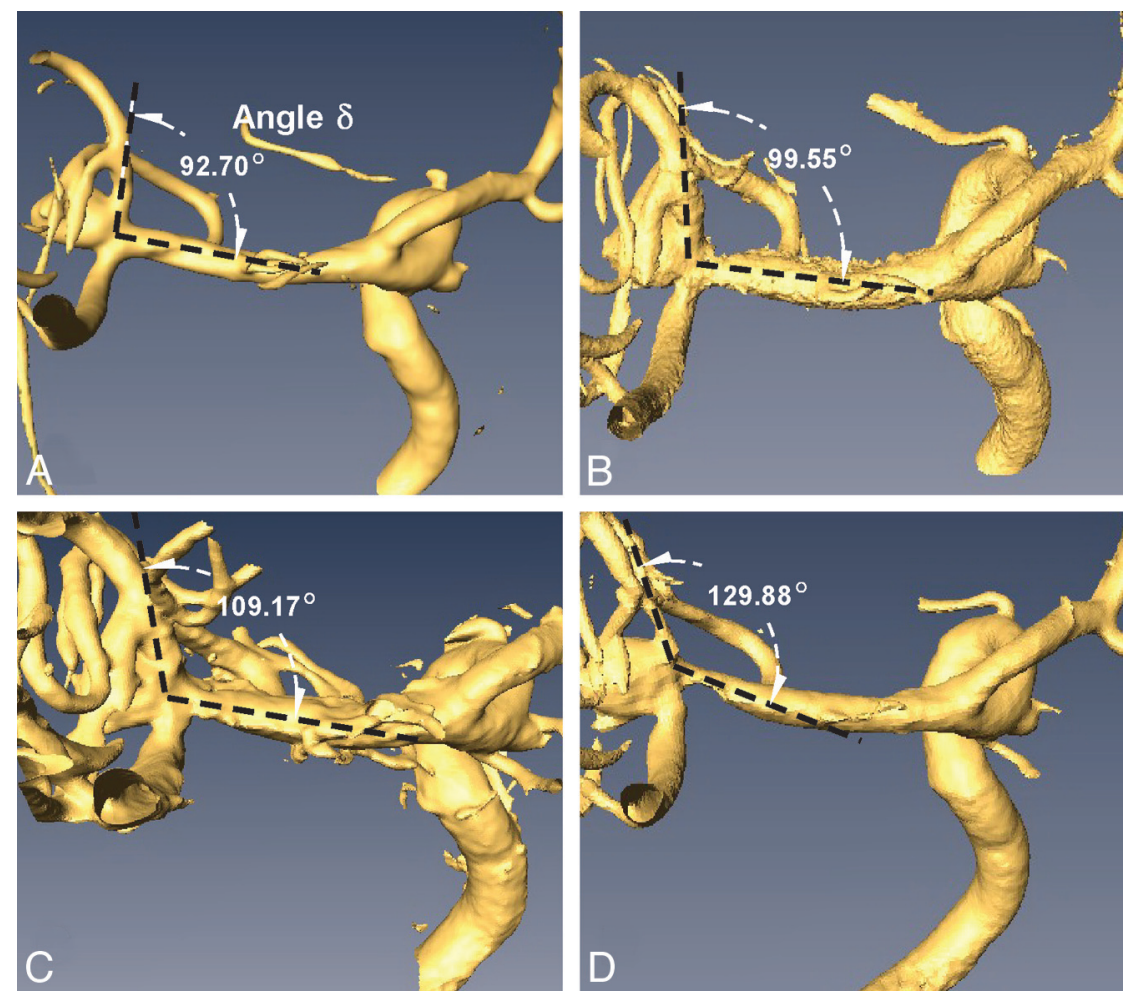

Fig 1. Volume rendered 3DRA datasets in a 67-year-old man with a wide-neck 7-mm MCA aneurysm show a pretreatment angle $\delta$ between the M1 and superior M2 segments of $92.7^{\circ}(A)$. which increased immediately to $99.6^{\circ}$ after stent coiling $(B)$, progressed to $109.2^{\circ}$ at 3 months $(C)$, and then to $129.9^{\circ}$ at 13 -month $(D)$ follow-up imaging.

\begin{tabular}{|c|c|c|c|}
\hline \multicolumn{4}{|c|}{$\begin{array}{l}\text { Angular alteration before and after stenting and at latest follow-up } \\
\text { and prestenting proximal vessel diameter (mean } \pm \text { SEM) }\end{array}$} \\
\hline $\begin{array}{l}\text { Bifurcation } \\
\text { Aneurysm } \\
\text { Location }\end{array}$ & $\begin{array}{l}\text { Prestenting } \\
\text { Angle } \delta\end{array}$ & $\begin{array}{l}\text { Post-Stent- } \\
\text { Coiling } \\
\text { Angle } \delta\end{array}$ & $\begin{array}{l}\text { Latest } \\
\text { Follow-Up } \\
\text { Angle } \delta\end{array}$ \\
\hline AcomA & $104.4 \pm 8.8^{\circ}$ & $131.5 \pm 5.3^{\circ a}$ & $148.8 \pm 7.7^{\circ \mathrm{b}}$ \\
\hline MCA & 105.4 & $116.8 \pm 6.6^{\circ \mathrm{C}}$ & $140.4 \pm 6.5^{o b}$ \\
\hline BA & $102.2 \pm 5.7^{\circ}$ & $120.7 \pm 5.2^{o d}$ & $132.6 \pm 4.3^{\circ \mathrm{e}}$ \\
\hline ICA & $97.4 \pm 29.3^{\circ}$ & $104.1 \pm 27^{\circ}$ & $119.6 \pm 26.1^{o b}$ \\
\hline Total & $103 \pm 4.2^{\circ}$ & $121 \pm 3.9^{\text {od }}$ & $137.3 \pm 3.9^{\circ e}$ \\
\hline
\end{tabular}

${ }^{a} P<.01$, compared with prestenting angle.

b $P<.01$, compared with post-stent angle.

$P<.05$, compared with prestenting-coiling angle.

$P<.0001$, compared with prestenting angle.

e $P<.0001$, compared with post-stent-coiling angle.

pre- and posttreatment) for a total mean difference of $34.3^{\circ}$. Analysis of the early (1-6 months) follow-up compared with immediate posttreatment revealed a greater increase of $14.5^{\circ}$ $(P<.0001)$ than the subsequent increase of just $5.8^{\circ}(P<$ $.0005)$ between the early and late follow-up ( $>6$ months), suggesting an asymptotic steady-state.

\section{Dependence of Remodeling on Bifurcation Location and Vessel Diameter}

Examination of treatment-induced bifurcation remodeling was noted in various locations, including the BA complex, the middle cerebral bifurcation, and, to a lesser degree, the ICA. Analysis of the maximal angular difference between the pretreatment and latest angiographic follow-up (Fig $2 A$ and Table) revealed a rank order of angular change being greatest at the AcomA $\left(44.5 \pm 5.9^{\circ}, P<.0001\right)>\mathrm{MCA}\left(35.0 \pm 4.0^{\circ}, P<\right.$ $.0001)>\mathrm{BA}\left(30.4 \pm 3.2^{\circ}, P<.0001\right)>\operatorname{ICA}\left(22.2 \pm 3.3^{\circ}, P=\right.$ $.021)$
Given this location-dependent change, we measured the diameters of the proximal (mother vessel) and distal (daughter branch) vessels undergoing stent deployment. This showed the proximal diameters of the ICA bifurcation to be greatest $(3.8 \pm 0.2 \mathrm{~mm}, P<.0001)$, followed by the MCA $(2.7 \pm 0.08$ $\mathrm{mm})$ and $\mathrm{BA}(2.7 \pm 0.08 \mathrm{~mm})$ bifurcations, with the AcomA having the smallest diameters $(2.2 \pm 0.15 \mathrm{~mm}, P<.01)$. The distal diameter of the stented vessel segment before stent placement was also largest for the ICA, followed by the MCA, AcomA, and BA, with a highly significant difference $(P<.01)$ between the ICA and BA or AcomA, and significant difference $(P<.05)$ between ICA and MCA bifurcations. No difference existed for the other pairs of vessels. Analysis of the proximal diameter of the stented-vessel segment with respect to the angular difference in $\delta$ between pre- and immediately posttreatment demonstrated an inverse relationship $(r=0.48, P<$ $.007)$ : the smaller the proximal vessel diameter, the bigger the angular increase caused by stent placement (Fig $2 B$ ). The same trend was noted, though not significantly, when evaluating the long-term angular change with respect to proximal vessel diameter $(r=0.117, P=.060)$. Analysis of the distal diameter of the stented-vessel segment versus the angular difference between prestenting and immediately after stent coiling demonstrated no significant relationship $(r=0.22, P=.22)$.

\section{Dependence of Angular Change on Pretreatment Angle}

We hypothesized that the factor responsible for the angular remodeling was the bending force $\left[\mathrm{F}_{\text {bending }}(\theta)\right]$ exerted by the deployed stent, which is dependent on the bending angle $(\theta=$ $180-\delta$ ) (Fig $2 C$ ). Analysis of the prestenting bending angle versus the angular change between pre- and poststenting revealed a significantly inverse relationship (Fig $2 D)(r=0.411, P=$ 


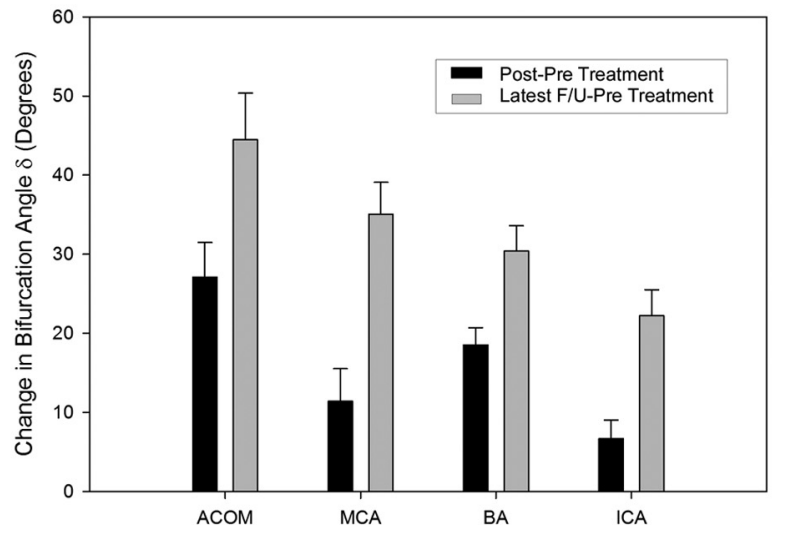

A

Stented Bifurcation Aneurysm Location

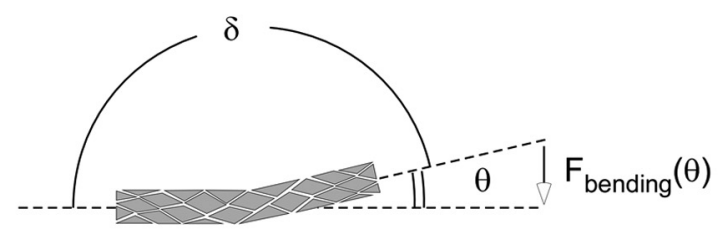

C
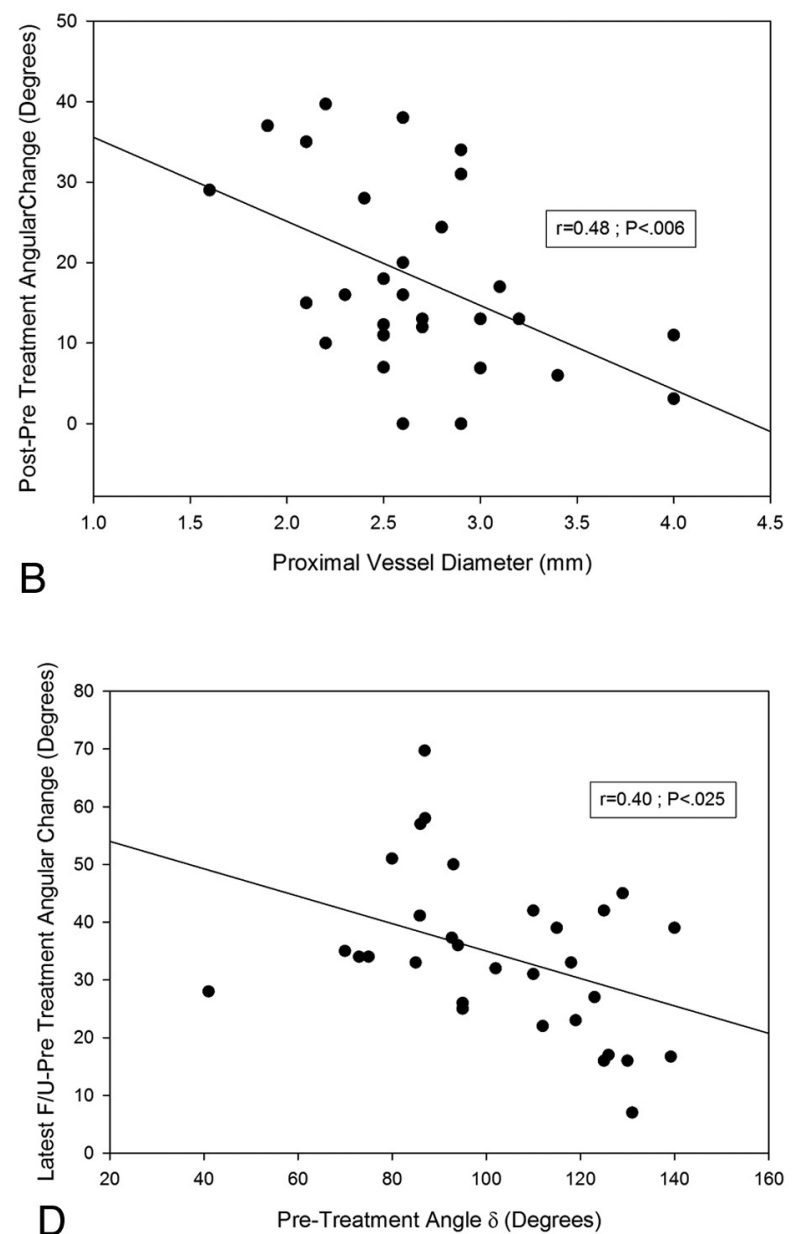

Fig 2. $A$, Dependence of immediate and delayed stent-induced angular remodeling on bifurcation location. $B$, An inverse linear relationship between the proximal vessel diameter and the stent-induced change in angle $\delta$. $C$, The relationship between the angle $\delta$, the bending angle $\theta\left(\theta=180^{\circ}-\delta\right)$, and the stent-reactive force $F_{\text {bending }}(\theta)$. $D$, The inverse linear dependence of ultimate angular remodeling on the pretreatment angle $\delta$, suggesting a link to the stent bending force.

.021), suggesting a link to the amount of stent deflection. The location dependence of the angular change, however, could not be solely accounted for by differences in the pretreatment angle because the latter was not significantly different among the 4 locations (Table).

\section{Longitudinally Stiffer Enterprise Stent Results in Greater Angular Remodeling}

We estimated the longitudinal stiffness of both types of stents and found the Enterprise stent to generate significantly greater tip force across the tested range of bending angles $\theta$ (Fig 3A), with linear least-squares fitting yielding a $70 \%$ greater slope coefficient $(0.026$ versus $0.015, P<.001)$. Enterprise cases showed greater bifurcation angular remodeling at latest follow-up with greater increase in angle $\delta$ compared with Neuroform stents $\left(39.2^{\circ}\right.$ versus $28.3^{\circ}, P=.028$ ) (Fig $3 B$ ). A similar but nonsignificant trend was noted when assessing immediate angular change at the time of the procedure $\left(20.8^{\circ}\right.$ versus $\left.14.6^{\circ}, P=.138\right)$. No significant differences were noted among Enterprise and Neuroform cases in proximal vessel diameter $(P=.302)$ or pretreatment angle $(P=.166)$.

\section{Discussion}

Bifurcation stent coiling results in a decrease of the effective neck and straightening of the vascular divider angle, which may effectively convert the morphology of a bifurcation aneurysm closer to that of a sidewall type. This study delineates the presence of a previously undefined immediate and delayed effect of stent deployment on the angular configuration of vascular bifurcations undergoing stent-mediated coiling by using self-expanding intracranial microstents. Previous studies have described the effect of balloon inflation-induced straightening of vessels during balloon-mounted stent deployment, ${ }^{10,11}$ with Zenteno et $\mathrm{al}^{10}$ suggesting that the resulting change in angulation of the parent artery could facilitate thrombosis of the aneurysm. Nonetheless, those results differ from the remodeling phenomenon reported here, which was seen in self-expanding stents that have a lower radial force and are deployed without use of balloon inflation. Furthermore, unlike the current study that involved the evaluation of bifurcation aneurysms, Zenteno et al mainly studied sidewall and fusiform aneurysms off a parent nonbifurcating vessel.

The Neuroform and Enterprise stents used in this study are both flexible and self-expanding nitinol stents specially manufactured for use in the cerebral vasculature..$^{5,6,8,26}$ The deployment of the stents in the vessels at the bifurcation was noted in itself to significantly modify the vascular angles as demonstrated at the end of the stent-coiling procedure. The extent of the vascular modification was inversely related to the vessel proximal diameter and pretreatment angle $\delta$. The great- 


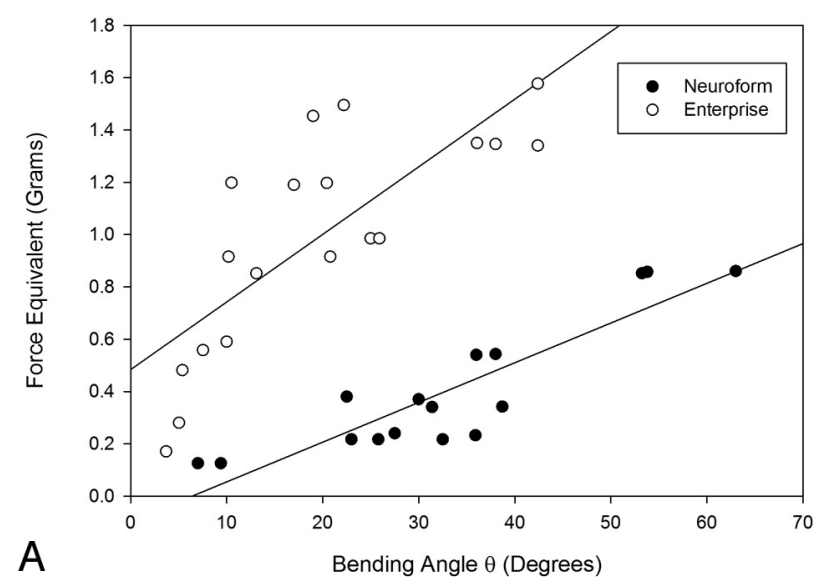

Neuroform: Force $=-0.0972+\left(0.0152{ }^{*} \theta\right) r=.89 p<.001$

Enterprise: Force $=0.484+(0.0259 * \theta) r=.78 p<.001$

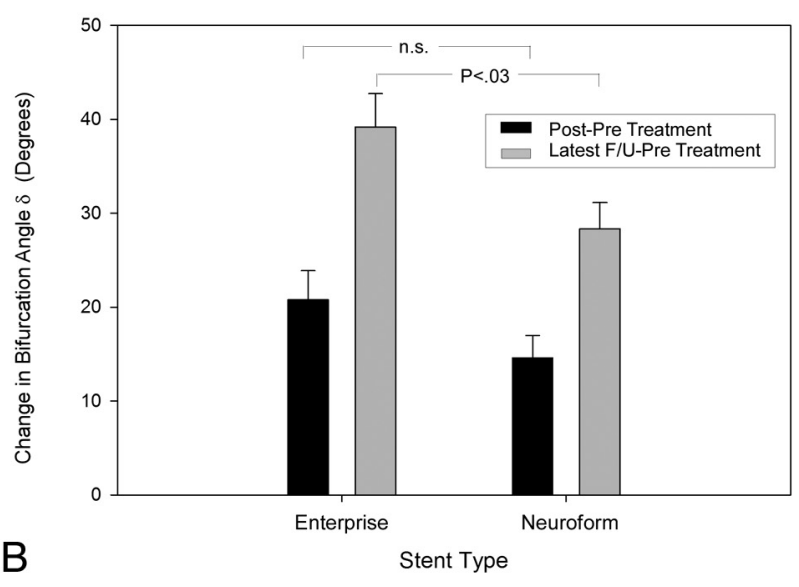

Fig 3. Stent reactive force $F_{\text {bending }}(\theta)$ (expressed in force-equivalent grams) $(A)$ in the Neuroform and Enterprise stents showing greater stiffness across the tested range in the closed-cell-design Enterprise device, along with a greater ultimate angular remodeling $(B)$ at latest-follow-up imaging

est angular remodeling was seen at the AcomA, followed by the BA and MCA, then by the ICA bifurcations, contrary to the order of the vessel caliber at these locations. This finding suggests that the greater the vessel size is, the greater is the resistance of the vessel against the straightening force exerted by the bent stent $\left(\mathrm{F}_{\text {bending }}\right)$. The smaller the prestenting vascular angle $\delta$ is, the greater is the deformation of the stent and its bending angle $\theta$ and consequently the greater is the straightening force of the stent, which causes greater angular modification following stent placement. This finding was also corroborated by the greater remodeling seen in the Enterprise stent subset, which was found to have a greater longitudinal stiffness and greater reactive force to bending (Fig $3 A$ ). However, at the time these procedures were performed, we were not aware of the difference in the longitudinal stiffness between the Enterprise and the Neuroform stents, and the finding was made retrospectively after we noticed a few cases of significant vessel straightening with the Enterprise stent.

With the passage of time, the persistent self-straightening tendency of these self-expanding shape-memory alloy stents was continually exerted on the cerebral vasculature, leading to the observed longer-term delayed greater angular expansion and vascular remodeling. Nonetheless, this phenomenon was noted to be more prominent during the earlier time periods (1-6 months) of follow-up, reaching steady-state subsequently, because the potential energy was mostly released by the stent, since the latter reverted to a shape closer to its preferred native straight configuration. Nevertheless, the latefollow-up remodeling of the stented angle was still significantly different $(P<.05)$ compared with immediate poststenting angles. It can be imagined that when the elasticity of the metal stent is fully released and the straightening force of the stent and the resistance exerted by the 3D structure of the cerebral vasculature reach a balance, the vascular angle will have reached a steady-state (Fig 1).

The apex of bifurcations is the site of maximum hemodynamic stress in a vascular network because of the direct impact, deflection, and separation of the blood flow streamlines and vortex formation at the lateral angles. ${ }^{27,28}$ The layer with the highest velocity of blood flow moves toward and directly impinges at the bifurcation apex where blood flow is divided. Thus, the arterial bifurcation apex experiences highly variable regions of wall shear stress, characteristic of flow separation. Regions of elevated shear stress are believed to cause injury to the endothelial cells of the vessel wall and predispose the vessel to diseases. ${ }^{27,29,30}$ The bifurcation angle may affect disease formation at the apex by influencing the tensile or stretching forces at the arterial bifurcation. ${ }^{31}$ The bifurcation angle may affect the formation of flow turbulence near the bifurcation apex. $^{32}$

After studying the characteristics of aneurysms on the ICA bifurcation, Sakamoto et al $^{31}$ reported that all the ICA bifurcation aneurysms deviated to the side of the A1 segment of the anterior cerebral artery, which formed a smaller angle with the ICA than that formed between the MCA and the ICA. Their result suggested higher hemodynamic stress experienced on the side of the Al segment. Aneurysm formation might be related to branching characteristics that locally increase the hemodynamic stresses, ${ }^{33,34}$ and normal cerebrovascular geometry may be a risk factor in this context.

The studies by Rossitti and Lofgren ${ }^{28,35}$ demonstrated that the branching angles of cerebral arteries may vary widely and that the apex of the bifurcation may lie in a nonoptimal position relative to the dividing streamline of the flow in the parent vessel, resulting in turbulence, vibrations, and increased shear stress on the vessel wall at the apical region, despite the fact that the blood flow/vessel radius relation is optimal. If aneurysm initiation or progression is related to the bifurcation angle at the vascular divider, then the balance of hemodynamic forces responsible likely will be altered by the stent-induced remodeling described in the current study. Furthermore, the angular remodeling properties of self-expanding stents, suggested to be at least partly the result of longitudinal stiffness in the current report, should be taken into consideration when evaluating stent design performance.

However, at the current stage, we are not sure what hemodynamic effect is induced by the angular remodeling caused by the longitudinal stiffness of the self-expanding stent. On one hand, this effect may be beneficial in the case of converting an aneurysm from bifurcation to sidewall because the flow would be diverted away from the aneurysm neck. On the other hand, 
it may be detrimental if a bifurcation aneurysm is converted into an endwall aneurysm such as may occur in the use of the waffle-cone technique. These theoretic hypotheses have to be both confirmed by hemodynamic modeling and, then, have to be corroborated clinically with long-term angiographic outcomes.

One additional point of concern raised by the angular remodeling described here relates to the theoretic possibility that the shape change may result in a relative movement of the coil mass, which could slowly impinge on the other branches at the vascular divider, possibly leading to delayed occlusion; this phenomenon was not witnessed in any of the cases herein.

\section{Conclusions}

This is the first study documenting a delayed remodeling process of cerebral vessels to the straightening force of self-expanding intracranial stents. Moreover, the smaller the native vessel or the prestenting vascular angle is, the bigger is the angular modification immediately following stent placement. This angular remodeling of the bifurcation may lead to possible alteration of hemodynamics at the vessel divider, an effect that requires further study. Clinical practitioners of intracranial stent placement should be aware of the delayed effect of stent placement at bifurcation aneurysms and its potential consequences on the relative position of the coil mass, both immediately postprocedure and its implications downstream. Stent manufacturers should also consider the long-term effect of longitudinal stiffness on the observed early and delayed cerebrovascular angular remodeling process.

Disclosures: Adel M. Malek has received research support from Codman Neurovascular and Boston Scientific, unrelated to the current study.

\section{References}

1. Alfke K, Straube T, Dorner L, et al. Treatment of intracranial broad-neck aneurysms with a new self-expanding stent and coil embolization. AJNR Am J Neuroradiol 2004;25:584-91

2. Benitez RP, Silva MT, Klem J, et al. Endovascular occlusion of wide-necked aneurysms with a new intracranial microstent (Neuroform) and detachable coils. Neurosurgery 2004;54:1359-67, discussion 1368

3. Biondi A, Janardhan V, Katz JM, et al. Neuroform stent-assisted coil embolization of wide-neck intracranial aneurysms: strategies in stent deployment and midterm follow-up. Neurosurgery 2007;61:460 - 68, discussion 468-69

4. Fiorella D, Albuquerque FC, Deshmukh VR, et al. Usefulness of the Neuroform stent for the treatment of cerebral aneurysms: results at initial (3-6-mo) follow-up. Neurosurgery 2005;56:1191-201, discussion 1201-02

5. Fiorella D, Albuquerque FC, Han P, et al. Preliminary experience using the Neuroform stent for the treatment of cerebral aneurysms. Neurosurgery 2004; 54:6-16, discussion 16-17

6. Lylyk P, Cohen JE, Ceratto R, et al. Endovascular reconstruction of intracranial arteries by stent placement and combined techniques. J Neurosurg 2002; 97:1306-13

7. Sani S, Lopes DK. Treatment of a middle cerebral artery bifurcation aneurysm using a double Neuroform stent "Y" configuration and coil embolization: technical case report. Neurosurgery 2005;57:E209, discussion E209

8. Bendok BR, Parkinson RJ, Hage ZA, et al. The effect of vascular reconstruction device-assisted coiling on packing density, effective neck coverage, and angiographic outcome: an in vitro study. Neurosurgery 2007;61:835-40, discussion $840-41$

9. Brassel F, Rademaker J, Haupt C, et al. Intravascular stent placement for a fusiform aneurysm of the posterior cerebral artery: case report. Eur Radiol 2001;11:1250-53

10. Zenteno MA, Murillo-Bonilla LM, Guinto G, et al. Sole stenting bypass for the treatment of vertebral artery aneurysms: technical case report. Neurosurgery 2005;57:E208, discussion E208

11. Zenteno MA, Santos-Franco JA, Freitas-Modenesi JM, et al. Use of the sole stenting technique for the management of aneurysms in the posterior circulation in a prospective series of 20 patients. J Neurosurg 2008;108:1104-18

12. Henkes H, Kirsch M, Mariushi W, et al. Coil treatment of a fusiform upper basilar trunk aneurysm with a combination of "kissing" Neuroform stents, TriSpan-, 3D- and fibered coils, and permanent implantation of the microguidewires. Neuroradiology 2004;46:464-68. Epub 2004 Apr 22

13. Benndorf G, Herbon U, Sollmann WP, et al. Treatment of a ruptured dissecting vertebral artery aneurysm with double stent placement: case report. AJNR Am J Neuroradiol 2001;22:1844-48

14. Doerfler A, Wanke I, Egelhof T, et al. Double-stent method: therapeutic alternative for small wide-necked aneurysms: technical note. J Neurosurg 2004;100: $150-54$

15. Chow MM, Woo HH, Masaryk TJ, et al. A novel endovascular treatment of a wide-necked basilar apex aneurysm by using a Y-configuration, double-stent technique. AJNR Am I Neuroradiol 2004;25:509-12

16. Thorell WE, Chow MM, Woo HH, et al. Y-configured dual intracranial stentassisted coil embolization for the treatment of wide-necked basilar tip aneurysms. Neurosurgery 2005;56:1035-40, discussion 1035-40

17. Horowitz M, Levy E, Sauvageau E, et al. Intra/extra-aneurysmal stent placement for management of complex and wide-necked- bifurcation aneurysms: eight cases using the waffle cone technique. Neurosurgery 2006;58:ONS-25862, discussion ONS-262

18. Albuquerque FC, Gonzalez LF, Hu YC, et al. Transcirculation endovascular treatment of complex cerebral aneurysms: technical considerations and preliminary results. Neurosurgery 2011;68:820-30

19. Cross DT 3rd, Moran CJ, Derdeyn CP, et al. Neuroform stent deployment for treatment of a basilar tip aneurysm via a posterior communicating artery route. AJNR Am J Neuroradiol 2005;26:2578-81

20. Fitzpatrick D, Chen M, Meyers PM. Horizontal Neuroform stent deployment for a ruptured basilar terminus aneurysm via the posterior communicating artery. J Vasc Interv Radiol 2006;17:1687-91

21. Fiorella D, Albuquerque FC, Woo H, et al. Neuroform in-stent stenosis: incidence, natural history, and treatment strategies. Neurosurgery 2006;59:34-42, discussion 34-42

22. Dhar S, Tremmel M, Mocco J, et al. Morphology parameters for intracranial aneurysm rupture risk assessment. Neurosurgery 2008;63:185-96, discussion 196-97

23. Baharoglu MI, Schirmer CM, Hoit DA, et al. Aneurysm inflow-angle as a discriminant for rupture in sidewall cerebral aneurysms: morphometric and computational fluid dynamic analysis. Stroke 2010;41:1423-30

24. Gao B, Malek AM. Possible mechanisms for delayed migration of the closed cell-designed Enterprise stent when used in the adjunctive treatment of a basilar artery aneurysm. AJNR Am J Neuroradiol 2010;31:E85-86

25. Bescos JO, Slob MJ, Slump CH, et al. Volume measurement of intracranial aneurysms from $3 \mathrm{D}$ rotational angiography: improvement of accuracy by gradient edge detection. AJNR Am J Neuroradiol 2005;26:2569-72

26. Weber W, Bendszus M, Kis B, et al. A new self-expanding nitinol stent (Enterprise) for the treatment of wide-necked intracranial aneurysms: initial clinical and angiographic results in 31 aneurysms. Neuroradiology 2007;49:555-61

27. Hademenos GJ, Massoud TF. Biophysical mechanisms of stroke. Stroke 1997; 28:2067-77

28. Rossitti S, Lofgren J. Vascular dimensions of the cerebral arteries follow the principle of minimum work. Stroke 1993;24:371-77

29. Szymanski MP, Metaxa E, Meng H, et al. Endothelial cell layer subjected to impinging flow mimicking the apex of an arterial bifurcation. Ann Biomed Eng 2008;36:1681-89

30. Takeuchi S, Karino T. Flow patterns and distributions of fluid velocity and wall shear stress in the human internal carotid and middle cerebral arteries. World Neurosurg 2010;7:174-85, discussion e27. Epub 2009 Oct 24

31. Sakamoto S, Ohba S, Shibukawa M, et al. Characteristics of aneurysms of the internal carotid artery bifurcation. Acta Neurochir (Wien) 2006;148:139-43, discussion 143

32. Roach MR, Scott S, Ferguson GG. The hemodynamic importance of the geometry of bifurcations in the circle of Willis (glass model studies). Stroke 1972;3:255-67

33. Stehbens WE. Etiology of intracranial berry aneurysms. J Neurosurg 1989;70: 823-31

34. Steiger HJ. Pathophysiology of development and rupture of cerebral aneurysms. Acta Neurochir Suppl (Wien) 1990;48:1-57

35. Rossitti S, Lofgren J. Optimality principles and flow orderliness at the branching points of cerebral arteries. Stroke 1993;24:1029-32 